\title{
Laboratory-Acquired Brucella Infection and S2 Vaccine infection Events in China
}

\author{
xiong zhu ${ }^{1}$, zhongzhi zhao ${ }^{1}$, chengling wang ${ }^{1}$, hai chen $^{1}$, miao wang ${ }^{1}$, kongjiao wei $^{1}$, \\ zhenjun $\mathrm{li}^{1}$, and Zhiguo Liu ${ }^{2}$ \\ ${ }^{1}$ Affiliation not available \\ ${ }^{2}$ National Institute of Infectious Diseases Control and Prevention, Chinese Center for \\ Disease Control and Prevention, Beijing 102206, China
}

August 23, 2020

\begin{abstract}
We report 10 laboratory-acquired Brucella infection cases and two S2 vaccine outbreak infection events during 2006-2016. Most laboratory-acquired Brucella infections were found in southern China, an emerging area of brucellosis. Microbiologists had a higher risk of laboratory-acquired Brucella infections, and the identification and handling of samples suspected of Brucella infection without recommended biosafety protection was the main reason for the infection. However, the S2 vaccine outbreak infection events were mainly observed in northern China, historically an area of human and animal brucellosis. The majority of infection cases were found among grassroot veterinarians and workers in animal vaccine production. Therefore, we suggest that identified suspected strains in biosafety facilities (BSL-3 and BSC) and abide biosafety practices are compulsory in southern, moreover, strengthening the preventive awareness of the clinical laboratory staff and physicians. In contrast, improving personal protection and launch biosafety education to high-risk of population exposure to S2 vaccine in northern is recommended.
\end{abstract}

\section{Introduction}

Brucellosis is one of the most common zoonotic diseases globally (Dean, Schelling, \& Zinsstag, 2014). More than 500,000 new human cases of brucellosis are reported annually (Deng, Liu, Duan, \& Peng, 2019), and millions of livestock are either infected or at risk (Pappas, 2010). It is a significant public health problem and causes serious harm to the development of the livestock industry and human health (Saddique et al., 2019). Brucella is most commonly transmitted by indirect or direct contact with infected livestock and the consumption of contaminated raw milk or their products (Kairu-Wanyoike et al., 2019). At present, 12 species have been identified in Genus Brucella, including six classical species and six newly detected biovars. Among them, Brucella melitensis is the most severe both in infectiousness and pathogenicity to humans (Eschenbrenner et al., 2002). Fever, fatigue, sweating, and muscle and joint pain are the main manifestations in the acute stage of human brucellosis (W. Jiang et al., 2019). The pathogen can invade various systems of the human body and seriously affect the quality of life (Venyo, 2015).

Brucella spp. are highly infectious because the infectious dose by an aerosol is only 10 to 100 organisms (D. L. Sewell, 2006), and it has been considered the most important laboratory-acquired bacterial infection (Al Dahouk \& Nöckler, 2011). Aerosol transmission generated accidentally or during microbiologic techniques from contaminated materials are the proposed routes of transmission (Bossi et al., 2004; Lowe et al., 2015). As aerosolization is the primary mechanism of transmission in this setting (Memish \& Mah, 2001), it is recommended that the organism be handled according to level 3 biosafety precautions (Fiori, Mastrandrea, Rappelli, \& Cappuccinelli, 2000). These guidelines can be challenging to follow, particularly in regions with a low incidence of brucellosis. All the Brucella spp. have been implicated in laboratory-associated infections, 
and they may account for up to $2 \%$ of all laboratory-associated infections (Robichaud, Libman, Behr, \& Rubin, 2004). In China, several cases of brucellosis are reported annually, but no national surveillance system specifically identifies laboratory-acquired cases. Therefore, the epidemiological profile of brucellosis resulting from laboratory transmission and S2 vaccine outbreak events of brucellosis in China remains unknown. The purpose of this study is to summarize laboratory-acquired infection and S2 vaccine outbreak events of brucellosis in China so that effective countermeasures for the prevention of laboratory-acquired infections and the promotion of public health safety can be designed.

\section{Material and Methods}

\section{Ethics Statement}

This study was carried out according to the principles of the Declaration of Helsinki. This study is a retrospective analyzed of historical documents collections and the research protocol was approved by the Ethics Committees of the National Institute for Communicable Disease Control and Prevention and the Chinese Center for Disease Control and Prevention.

\section{Data Source}

The source of cases of laboratory-acquired infection was the Chinese academic database: CNKI (www.cnki.net), Wanfang (www.wanfangdata.com.cn). Similarly, the reported S2 vaccine outbreak events were collected in this academic database and also the internet. The search terms were "Brucella," "brucellosis," "laboratory-acquired infection" or "hospital-acquired infection," S2 vaccine infection events; Search field: Keyword, Abstract, and full article; search language: Chinese; the retrieval time: from the construction of the databases to June 2, 2020. Excel 2016 (Microsoft, Redmond, WA, USA) was used for data processing and analysis.

\section{Results}

\section{Laboratory-acquired Infections}

A total of nine laboratory-acquired infection events were reported during the 2006-2016 period in China (Table. 1). Two events were observed in northern China (Beijing and Ningxia province), and the remaining seven events were found in southern China (Zhejiang, Jiangsu, Jiangxi, and Anhui provinces). Seven of them were microbiological technicians, and the other two were cleaners of a microbiology laboratory. The age range of the nine cases was 28-65, and the mean age of 45 . Two were males, and seven were females. Five patients were infected by identifying or handling suspect Brucella strains, two by handling blood culture samples from patients with brucellosis, and the remaining individuals were infected while cleaning up the microbiology laboratory waste. All accidental infections occurred due to substandard laboratory safety conditions, manipulations outside biological safety cabinets, or inadequate personal protective equipment. Fever was the most common clinical manifestation among patients, who also suffered from fatigue, sweating, joint pain, and headache. Only one patient was suspected of brucellosis; seven cases were diagnosed with a fever of unknown origin, and one patient was diagnosed with inflammation of the psoas major muscle. Eight patients were first diagnosed with brucellosis by bacteriology test, and a serology test (SAT titer[?]1:100) was used only in one case.

From March to May 2011, a total of 28 individuals (including 27 students and one teacher) were diagnosed with Brucella infections in the college of animal medicine of Northeast Agricultural University. The field epidemiology survey showed that the source of infection were four goats without the quarantine inspection that experimental animals require. In December 2010, teachers of the College of Animal Medicine of Northeast Agricultural University purchased four goats from a Qingxi farm, Jijia village, Xingfu Town, Xiangfang District, Harbin city. However, they did not follow the quarantine and inspection regulations on the management of experimental animals of the national and Heilongjiang Province. Subsequently, the four goats infected withBrucella were used for teaching experiments (obstetric experiment and animal anatomy experiment) five times, involving four teachers, two experimenters, and 110 students, resulting in the brucellosis infections in 27 students and one teacher in this college from March to May 2011. Based on a comprehensive 
survey and the analysis of the four goats, these animals (infected with brucellosis) were found to be the source of infection for these cases. Aerosols and contact with infected goats were the main route of transmission, and the failure to comply with the standard experimental norms and ensure that the students would follow the biosafety protection-operating procedures were the main reason for this cluster infection events. According to the unified prescription by the expert Committee on accident handling, the treatment consisted of intravenous doses of combined doxycycline, rifampin, and levofloxacin (or etoperidone sulbactam) for six weeks, followed by oral rifapentine and tetracycline for 40 days. Twenty-five patients were cured, one patient improved, and two patients still showed a small amount of effusion in the joint cavity by MRI.

\section{S2 Vaccine Infection Events}

In 2017, a brucellosis outbreak caused by S2 vaccination was reported in the Tianzhu county Gansu Province, China (Zhang P, 2018). An investigation showed that a total of 206 animal epidemic prevention controllers participated in the immunization work of S2 vaccination of sheep in the Tianzhu county from November to December in 2016. Fifty-one controllers were diagnosed with brucellosis by serology testing, and the rate of infection was $24.8 \%$. All 51 individuals were RBPT-positive, in 36 cases SAT [?] 1:100, the SAT ranged from 1: 50 to 1:100 in 15 cases. The blood samples from all patients were free of Brucellastrains. The vaccination work did not comply with the biosafety regulations was the main reason for this infection events, including improper handling in vaccination and limited understanding in pathogenicity of the $\mathrm{S} 2$ vaccine, inadequate personal protection, and imperfect emergency measures. The $94.13 \%$ cases (48/51) represent fatigue and sweat, fever in four cases, and swelling of testis in five cases. All the patients followed a unified therapeutic schedule of combined doxycycline and rifampin. The therapy was sustained for four weeks to six months based on the illness situation of patients. All the patients greatly improved. It is recommended that the animal husbandry and veterinary department should strengthen the protection knowledge of brucellosis and carry out vaccination according to the biological safety regulation.

On November 28, 2019, two students from the prevention and control technology team of Lanzhou Veterinary Research Institute of the Chinese Academy of Agricultural Sciences were found to have serum with antiBrucella antibodies. Up to December 25, 671 serum samples from students and staff of the Lanzhou Animal Research Institute were screened. It was found that 181 persons were positive for anti-Brucella antibodies, and only one person had clinical symptoms. Active, free, and standardized treatment was carried out in patients who voluntarily agreed. Based on the investigation and many field test results, the expert concluded that from July 24 to August 20, 2019, the expired disinfectant was used in the production process of the S2 vaccine for animal use by Lanzhou biopharmaceutical factory of China animal husbandry, which resulted in incomplete sterilization of the waste gas from the production fermentation tank. The waste gas carrying the fermentation liquid formed the bacterial aerosols thought to be the main reason for this outbreak event. During the production period, the main wind direction in this area is the southeast wind, and the Lanzhou Veterinary Institute is located in the downwind direction of the Lanzhou biological pharmaceutical factory of China animal husbandry. Individuals become seropositive when they inhale or have mucous membrane contacts with infectious bacteria. The government sector has instructed the Zhongmu Lanzhou biopharmaceutical plant to focus on the brucellosis vaccine workshop to immediately carry out a comprehensive inspection on the implementation of the quality management specified for veterinary medicine in the whole plant, and to make the proper rectifications within a time limit. Without prior acceptance of the competent department of the industry, the brucellosis vaccine workshop should not resume production.

\section{Discussion}

Brucellosis is the most common bacterial laboratory-acquired infection worldwide, owing in part to the low infectious dose and high attack rate following a laboratory exposure (Blaney, Bosserman, Bui, Guerra, \& Stoddard, 2009). It is also easily aerosolized and has the potential to cause acute and chronic illness (D L Sewell, 1995). Humans are usually infected by occupational exposure to infected animals, the consumption of unpasteurized dairy products from infected animals, or inhalation of infectious aerosols. Clinical laboratory workers have failed to recognize suspicious isolates and have manipulated unknown isolates on open benches, using procedures that could aerosolize brucellae, increasing their exposure risk to biology hazards 
(Ackelsberg et al., 2020). In the present study, eight laboratory-acquired infection events were found in the southern region, a brucellosis emerging area (Beijing city). Another event was observed in Ningxia province, which is historically an area of brucellosis. In the past, more than $90 \%$ of human brucellosis cases in China appeared in the northern animal husbandry provinces (Shang, Xiao, \& Yin, 2002). However, since 2010, human brucellosis has occurred or reappeared in all provinces in southern China (Yang et al., 2020). Southern China is an emerging area of brucellosis with the lowest incidence of brucellosis, but the incidence is increasing (Yang et al., 2020). In the southern regions, clinical microbiology laboratories are frequently unfamiliar with the genus of Brucella, and there is a low index of suspicion by physicians or failure to notify the laboratory that the handled specimens might yield a hazardous organism. Typically, clinicians do not consider brucellosis until notified that bacteremia with Brucella was suspected. Moreover, the misidentification of the organism by commercial systems, unsafe laboratory practices, and laboratory accidents have been responsible for many cases of exposure to the organism and laboratory-acquired disease in recent years (Yagupsky \& Baron, 2005). In the United States, fewer than 150 cases of brucellosis have been reported annually since 1986, but brucellosis is among the most commonly reported laboratory-acquired bacterial infections (Pike, 1976). In Indiana and Minnesota, two cases of brucellosis in microbiologists at two clinical laboratories were reported to state health departments in 2006. The results of that investigation showed that Brucella spp. pose a risk of aerosol-transmitted infection ("Laboratory-acquired brucellosis-Indiana and Minnesota, 2006," 2008). Unknown isolates manipulated and/or tested on open benches was the main risk factor in the laboratory-acquired infection of brucellosis. In this study, the problematic current practices when handling blood culture bottles from patients with fever of unknown origin were the main reason for these infectious events, and the transmission in our cases was probably due to aerosol contamination. Similarly, most critical exposures involved catalase testing or isolate vortexing, both of which may generate infectious aerosols (Stephanie et al., 2004). In New York City, during the 2015-2017 period, over 200 occupational exposures occurred when the unknown isolates were manipulated and/or tested on open benches, including procedures that could generate infectious aerosols (Ackelsberg et al., 2020). In Turkey, where biosafety cabinets do not exist in most hospital laboratories, many laboratory-acquired Brucella infection events have been reported (Sayin-Kutlu et al., 2012). In our study, we observed a high risk of developing laboratory-acquired brucellosis in microbiological laboratory workers. In Spain, a total of 75 works have suffered from laboratory-acquired brucellosis, $57 \%$ (43/75) of whom were microbiologists (Bouza, SanchezCarrillo, Hernangomez, \& Gonzalez, 2005). Thus, Brucella spp. have persisted as biological hazards to clinical laboratory workers manipulating unknown clinical isolates. This suggests that we should improve the ability of clinicians to diagnose brucellosis, especially in the southern region. Furthermore, it is essential to promote the use of biosafety level 3 (BSL-3) facilities when handling specimens from patients suspected of brucellosis infections and also unidentified isolates. Moreover, the following practices should be followed: timely exposure risk identification, information sharing, postexposure prophylaxis (PEP), and appropriate follow-up of potentially exposed workers, in combination with worker training to maximize awareness and observance of proper safety practices. Definitions for the laboratory exposure risk toBrucella spp. and recommendations for PEP have been developed and applied to the laboratory-acquired brucellosis cases that occurred in Indiana and Minnesota (Pien, Saah, Miller, \& Woods, 2006). Finally, we consider that the use of gloves, masks, goggles, and a biosafety cabinet, and also the continuous strengthening of the education on biosafety of individuals with high infection risk is significant for preventive laboratory-acquired brucellosis events.

The B. suis strain S2 is a widely used live vaccine in China, which was isolated from a swine fetus at the Institute of Inspection for Veterinary Medicine in 1952 (H. Jiang et al., 2018). A study suggested that the S2 vaccine is of low virulence, stimulates good humoral and cellular immunity, and protect animals against infection by heterologous, virulent Brucella species (Zhu et al., 2016). In the present study, more than 50 individuals infected in the context of S2 vaccination had clinical manifestations, and 180 persons had no clinical symptoms. Another study confirmed that the S2 vaccine had low residual virulence, and the immunity against the B. melitensis strain induced by S2 had declined after 150 days more than those of S19 and Rev.1 (Bosseray \& Plommet, 1990). The low residual virulence of S2 may be the main reason why the patients had no obvious clinical symptoms. Similarly, a B. abortus S19 vaccine strain was isolated from four 
persons; all four infected individuals were employed as milkers and did not have apparent manifestations of the disease (Osman, Hassan, Ali, Abdoel, \& Smits, 2015). Moreover, human illness associated with the vaccine strain RB51 has been documented from inadvertent needle sticks or inoculation of the conjunctiva or open wounds with RB51 (Ashford et al., 2004; Cossaboom et al., 2018; Hatcher et al., 2018). The first case of human brucellosis in Bosnia and Herzegovina was caused by live attenuated Brucella melitensis Rev.1 strain (Arapovic et al., 2020). Although the vaccine strain may be transmitted to the human population and may persist in the blood sometimes without causing overt disease, protective measures remain important to prevent exposure from vaccine strains. Moreover, more than 100 grassroots animal epidemic prevention officers from Ulanqab of Inner Mongolia were diagnosed as having brucellosis from short-time period, but the conventional serological tests used did not allow to distinguish between vaccine-associated infection and infections by contact with infected animals.

\section{Conclusion}

Laboratory testing and vaccination have played a crucial role in the prevention and control of brucellosis worldwide. Still, laboratory-acquired infection and vaccination-related infection events are worthy of attention. With the development of molecular biology techniques and a deep understanding of the pathogenic mechanism ofBrucella, new vaccines based on genome engineering are expected to replace traditional vaccines for brucellosis control. This report serves as a reminder that occupational Brucella exposure is a risk among clinical laboratory staff and veterinary personnel. Clinicians, laboratory staff members, and public health officials should be aware of diagnosis and treatment challenges of occupational brucellosis and be prepared to manage brucellosis occupational exposures and improve the awareness of brucellosis in high-risk populations. Only the implementation of these measures will eliminate this occupational infection.

\section{Abbreviation}

BSL3: Biosafety Level 3 (BSL-3)

BSC: Biosafety cabinet

PEP: postexposure prophylaxis

\section{Funding}

This study was supported by the biosafety Key Special Projects (2019YFC1200705), Major Special Projects for Infectious Disease (Nos. 2017ZX10303401, 2018ZX10734401, and 2018ZX10734404), and the Nature Science Fund of the Inner Mongolia Autonomous Region (No. 2018MS08004). The funding agencies had no role in the study design, data collection, and analysis, decision to publish, or preparation of the manuscript.

\section{Authors' Contributions}

ZX and LZG collected and classified documents. ZZZ and WKJ processed and analyzed the data. LZG and WM drafted the manuscript. WCL participated in the critical reviewing of the manuscript. ZX and LZJ participated in the design of the study and managed the project. All the authors read and approved the final version of the manuscript.

\section{Data Availability Statement}

All data generated or analyzed during this study are included in our article.

\section{Conflict of Interest}

No conflicts of interest were reported.

\section{References:}

Ackelsberg, J., Liddicoat, A., Burke, T., Szymczak, W. A., Levi, M. H., Ostrowsky, B., . . . Layton, M. (2020). Brucella Exposure Risk Events in 10 Clinical Laboratories, New York City, USA, 2015 to 2017. J. Clin. Microbiol., 58 (2). doi:10.1128/jcm.01096-19 
Al Dahouk, S., \& Nockler, K. (2011). Implications of laboratory diagnosis on brucellosis therapy. Expert Rev Anti Infect Ther, 9 (7), 833-845. doi:10.1586/eri.11.55

Arapovic, J., Špičić, S., Duvnjak, S., Ostojić, M., Arapović, M., Nikolić, J., \& Cvetnić, Ž. (2020). The first report of Brucella melitensis Rev.1 human brucellosis in Bosnia and Herzegovina. J Infect Dev Ctries, 14 (2), 232-235. doi:10.3855/jidc.11949

Ashford, D. A., di Pietra, J., Lingappa, J., Woods, C., Noll, H., Neville, B., . . . Perkins, B. A. (2004). Adverse events in humans associated with accidental exposure to the livestock brucellosis vaccine RB51. Vaccine, 22 (25-26), 3435-3439. doi:10.1016/j.vaccine.2004.02.041

Blaney, D., Bosserman, E., Bui, D., Guerra, M., \& Stoddard, R. (2009).Brucella Laboratory Exposures in the United States, 2008. Paper presented at the Infectious Diseases Society of America Meeting.

Bosseray, N., \& Plommet, M. (1990). Brucella suis S2, brucella melitensis Rev. 1 and Brucella abortus S19 living vaccines: residual virulence and immunity induced against three Brucella species challenge strains in mice. Vaccine, 8 (5), 462-468. doi:10.1016/0264-410x(90)90247-j

Bossi, P., Tegnell, A., Baka, A., van Loock, F., Hendriks, J., Werner, A., . . . Gouvras, G. (2004). Bichat guidelines for the clinical management of brucellosis and bioterrorism-related brucellosis.Euro Surveill., 9 (12), 33-34. doi:10.2807/esm.09.12.00506-en

Bouza, E., Sánchez-Carrillo, C., Hernangómez, S., \& González, M. J. (2005). Laboratory-acquired brucellosis: a Spanish national survey.J. Hosp. Infect., 61 (1), 80-83. doi:10.1016/j.jhin.2005.02.018

Cossaboom, C. M., Kharod, G. A., Salzer, J. S., Tiller, R. V., Campbell, L. P., Wu, K., . . Stonecipher, S. (2018). Notes from the Field: Brucella abortus Vaccine Strain RB51 Infection and Exposures Associated with Raw Milk Consumption - Wise County, Texas, 2017. MMWR Morb. Mortal. Wkly. Rep., 67 (9), 286. doi:10.15585/mmwr.mm6709a4

Dean, A., Schelling, E., \& Zinsstag, J. (2014). Brucellosis.Neglected Tropical Diseases .

Deng, Y., Liu, X., Duan, K., \& Peng, Q. (2019). Research Progress on Brucellosis. Curr. Med. Chem., 26 (30), 5598-5608. doi:10.2174/0929867325666180510125009

Eschenbrenner, M., Wagner, M. A., Horn, T. A., Kraycer, J. A., Mujer, C. V., Hagius, S., . . DelVecchio, V. G. (2002). Comparative proteome analysis of Brucella melitensis vaccine strain Rev 1 and a virulent strain, 16M. J. Bacteriol., 184 (18), 4962-4970. doi:10.1128/jb.184.18.4962-4970.2002

Fiori, P. L., Mastrandrea, S., Rappelli, P., \& Cappuccinelli, P. (2000). Brucella abortus infection acquired in microbiology laboratories. J. Clin. Microbiol., 38 (5), 2005-2006.

Hatcher, S. M., Shih, D., Holderman, J., Cossaboom, C., Leman, R., \& DeBess, E. (2018). Notes from the Field: Adverse Event Associated with Unintentional Exposure to the Brucella abortus RB51 Vaccine Oregon, December 2017. MMWR Morb. Mortal. Wkly. Rep., 67 (26), 747. doi:10.15585/mmwr.mm6726a4

Jiang, H., Dong, H., Peng, X., Feng, Y., Zhu, L., Niu, K., . . . Ding, J. (2018). Transcriptome analysis of gene expression profiling of infected macrophages between Brucella suis 1330 and live attenuated vaccine strain S2 displays mechanistic implication for regulation of virulence. Microb. Pathog., 119 , 241-247. doi:10.1016/j.micpath.2018.04.003

Jiang, W., Chen, J., Li, Q., Jiang, L., Huang, Y., Lan, Y., \& Li, Y. (2019). Epidemiological characteristics, clinical manifestations and laboratory findings in 850 patients with brucellosis in Heilongjiang Province, China. BMC Infect. Dis., 19 (1), 439. doi:10.1186/s12879-019-4081-5

Kairu-Wanyoike, S., Nyamwaya, D., Wainaina, M., Lindahl, J., Ontiri, E., Bukachi, S., . . Bett, B. (2019). Positive association between Brucella spp. seroprevalences in livestock and humans from a crosssectional study in Garissa and Tana River Counties, Kenya.PLoS Negl Trop Dis, 13 (10), e0007506. doi:10.1371/journal.pntd.0007506 
Laboratory-acquired brucellosis-Indiana and Minnesota, 2006. (2008).MMWR Morb. Mortal. Wkly. Rep., $57(2), 39-42$.

Lowe, C. F., Showler, A. J., Perera, S., McIntyre, S., Qureshi, R., Patel, S. N., . . Muller, M. P. (2015). Hospital-associated transmission of Brucella melitensis outside the laboratory.Emerging Infect. Dis., 21 (1), 150-152. doi:10.3201/eid2101.141247

Memish, Z. A., \& Mah, M. W. (2001). Brucellosis in laboratory workers at a Saudi Arabian hospital. Am J Infect Control, 29 (1), 48-52. doi:10.1067/mic.2001.111374

Osman, A. E., Hassan, A. N., Ali, A. E., Abdoel, T. H., \& Smits, H. L. (2015). Brucella melitensis Biovar 1 and Brucella abortus S19 Vaccine Strain Infections in Milkers Working at Cattle Farms in the Khartoum Area, Sudan. PLoS ONE, 10 (5), e0123374. doi:10.1371/journal.pone.0123374

Pappas, G. (2010). The changing Brucella ecology: novel reservoirs, new threats. Int. J. Antimicrob. Agents, 36 Suppl 1, S8-11. doi:10.1016/j.ijantimicag.2010.06.013

Pien, B. C., Saah, J. R., Miller, S. E., \& Woods, C. W. (2006). Use of sentinel laboratories by clinicians to evaluate potential bioterrorism and emerging infections. Clin. Infect. Dis., 42 (9), 1311-1324. doi:10.1086/503260

Pike, R. M. L. (1976). Laboratory associated infections: summary and analysis of 3921 cases. Health Lab Sci, 13 (2), 105-114.

Robichaud, S., Libman, M., Behr, M., \& Rubin, E. (2004). Prevention of laboratory-acquired brucellosis. Clin. Infect. Dis., 38 (12), e119-122. doi:10.1086/421024

Saddique, A., Ali, S., Akhter, S., Khan, I., Neubauer, H., Melzer, F., . . El-Adawy, H. (2019). Acute Febrile Illness Caused by Brucella abortus Infection in Humans in Pakistan. Int $J$ Environ Res Public Health, 16 (21). doi:10.3390/ijerph16214071

Sayin-Kutlu, S., Kutlu, M., Ergonul, O., Akalin, S., Guven, T., Demiroglu, Y. Z., . . . Akova, M. (2012). Laboratory-acquired brucellosis in Turkey. J. Hosp. Infect., 80 (4), 326-330. doi:10.1016/j.jhin.2011.12.020

Sewell, D. L. (1995). Laboratory-Associated Infections and Biosafety.Clinical Microbiology Reviews, 8 (3), 389-405.

Sewell, D. L. (2006). Laboratory-acquired infections: Are microbiologists at risk? Clin. Microbiol. Newsl., 28 (1), 1-6. doi:10.1016/j.clinmicnews.2005.12.004

Shang, D., Xiao, D., \& Yin, J. (2002). Epidemiology and control of brucellosis in China. Veterinary Microbiology, 90 (1-4), 165-182.

Stephanie, N., Richard, G., Molly, K., Limberger, R. J., Karen, D. A., Louise, C., . . Nellie, D. (2004). Laboratory-acquired Brucellosis.Emerging Infectious Diseases, 10 (10), 1848-1850.

Venyo, A. K. G. (2015). Brucellosis of testis and epididymis: a review of the literature. British Journal of Medicine 8 Medical Research .

Yagupsky, P., \& Baron, E. J. (2005). Laboratory exposures to brucellae and implications for bioterrorism. Emerging Infect. Dis., 11 (8), 1180-1185. doi:10.3201/eid1108.041197

Yang, H., Zhang, S., Wang, T., Zhao, C., Zhang, X., Hu, J., . . Z Zhen, Q. (2020). Epidemiological Characteristics and Spatiotemporal Trend Analysis of Human Brucellosis in China, 1950-2018. Int J Environ Res Public Health, 17 (7). doi:10.3390/ijerph17072382

Zhang P, F. C., Cui BY. (2018). A report of a brucellosis outbreak caused by vaccination. Disease Surveillance, 33(3) , 222-224. doi:10.3784/j.issn.1003-9961.2018.03.013

Zhu, L., Feng, Y., Zhang, G., Jiang, H., Zhang, Z., Wang, N., . . . Suo, X. (2016). Brucella suis strain 2 vaccine is safe and protective against heterologous Brucella spp. infections. Vaccine, 34 (3), 395-400. 
doi:10.1016/j.vaccine.2015.09.116

Table 1. Epidemiology and clinical diagnosis characteristics of patients with brucellosis

\begin{tabular}{llllll}
\hline Cases & Time & Location & Patient profiles & Transmission route & Main symptoms \\
\hline 1 & $2006-01$ & Beijing & Male, 45, laboratorian & Identification of suspect Brucella & Fever, fatigue, chills, headach \\
2 & $2007-09$ & Hangzhou & Female, 34, laboratorian & Handling of suspected samples & Undulant fever, fatigue, \\
3 & $2012-02$ & Suzhou & Female, 46, laboratorian & Identification of suspect Brucella & Fatigue, intermittent low feve \\
4 & $2012-05$ & & Female, 39, laboratorian & Handling a strain from case 3 & Fatigue, low fever, sweating, \\
5 & $2013-03$ & Ningxia & Female, 40, laboratorian & Identification of suspect Brucella & Fever of unknown, 39. $8^{\circ} \mathrm{C}$, su \\
6 & $2013-06$ & Yangzhou & Female, 49, laboratorian & Handling blood culture samples & Undulant fever, sweating \\
7 & $2016-10$ & Nanchang & Female, 60, cleaner & Cleaning up the laboratory waste & Fever, fatigue, sweating, lum \\
8 & & & Male, 65, cleaner & Cleaning up the laboratory waste & High fever of unknown origin \\
9 & $2016-11$ & Hefei & Female, 28, laboratorian & Handling blood culture samples & Undulant fever, sweating, joi \\
\hline
\end{tabular}

